\title{
Characterisation of Neisseria gonorrhoeae from women with simultaneous infections at two sites
}

\author{
ROBERT C NOBLE \\ From the Division of Infectious Diseases, Department of Medicine, University of Kentucky College of \\ Medicine, Lexington, Kentucky, USA
}

SUMMARY Infection with two strains of Neisseria gonorrhoeae was found in paired cultures from $8 \cdot 7 \%(6 / 69)$ of women who had infections of the cervix, rectum, or urethra. Paired gonococcal cultures from each patient were characterised by auxotyping, susceptibility to four antibiotics, and acrylamide gel electrophoresis patterns of the gonococcal cell proteins.

\section{Introduction}

Gonococci may be isolated simultaneously from more than one site in women with gonorrhoea when cultures are taken from the urethra, cervix, and rectum. ${ }^{1}$ Rein et $a l^{2}$ studied women with gonococcal infection of the rectum. In $40 \%$ of 3183 women treated for gonorrhoea, rectal infection accompanied cervical infection. Thirty-one per cent of women with infection of both cervix and rectum harboured different gonococcal strains on the basis of their different antibiotic susceptibilities. From Rein's ${ }^{2}$ collection of gonococci six paired isolates with different antibiotic susceptibilities also had different gonococcal auxotypes as well as different acrylamide gel electrophoresis patterns of the gonococcal cell proteins. ${ }^{3}$ These results suggested that possibly onethird of women with gonococcal infection of the cervix and rectum were infected with more than one strain of Neisseria gonorrhoeae.

The present study was designed to estimate the frequency with which different gonococcal strains were present at the same time in paired genitorectal cultures of women with gonorrhoea. Paired cultures were examined by means of gonococcal auxotyping, susceptibility to four antibiotics, and patterns produced by acrylamide gel electrophoresis of solubilised gonococcal cell proteins.

\section{Patients and methods}

BACTERIAL ISOLATES

The isolates were taken from 69 women (45 white, 23

Address for reprints: Dr R C Noble, Division of Infectious Diseases, Department of Medicine, University of Kentucky College of Medicine, Lexington, Kentucky 40536, USA

Received for publication 12 March 1979 black, 1 race unknown) with uncomplicated gonorrhoea attending the Lexington-Fayette County Health Department Venereal Disease Clinic. The patients were selected because at one clinic visit cultures giving positive results had been taken from two of the following sites: cervix, rectum, or urethra. The specimens were isolated on modified ThayerMartin medium and were identified by bacterial morphology, Gram stain, oxidase reaction $(1 \%$ solution of $\mathrm{N}, \mathrm{N}$-dimethyl-p-phenylenediamine monohydrochloride), and the production of acid with dextrose- but not maltose-, lactose-, sucrose-, or fructose-containing media. GC medium base (Difco Laboratories, Detroit, Michigan) with $1 \%$ IsoVitalex (Baltimore Biological Laboratory, Cockeysville, Maryland) was used as the culture medium for the study and for identification of the colonial morphological types. These were identified by observation of the bacterial colonies in indirect light. ${ }^{4}$ Studies of the paired isolates were performed on a single colonial type. Gonococcal isolates were stored at $-70^{\circ} \mathrm{C}$ in tryptic soy broth (Difco) containing $13 \%$ glycerol.

\section{ANTIBIOTIC SUSCEPTIBILITY TESTS}

Antibiotic susceptibility tests were performed using the plate dilution technique. ${ }^{5}$ Reference microorganisms with known antibiotic susceptibilities were included as controls in each test. The antibiotics (purchased from the United States Pharmacopeial Convention Inc., Bethesda, Maryland) were tested by means of two-fold dilutions in agar (ampicillin and penicillin $0 \cdot 007-2 \mu \mathrm{g} / \mathrm{ml}$, tetracycline $0 \cdot 09-25$ $\mu \mathrm{g} / \mathrm{ml}$, and spectinomycin $0 \cdot 39-100 \mu \mathrm{g} / \mathrm{ml}$ ). Antibiotic susceptibility results of paired isolates were judged to be alike if the test results were within two dilutions in the two isolates. 
GONOCOCCAL AUXOTYPING

Gonococcal auxotyping was performed by the method of Catlin' ${ }^{6}$ and Carifo and Catlin. ${ }^{7}$ Auxotyping was performed twice on each isolate and gonococci of a known auxotype were included in each test.

\section{ACRYLAMIDE GEL ELECTROPHORESIS}

Acrylamide gel electrophoresis of the cell proteins of the gonococcal isolates was performed as previously described. ${ }^{3}$ Overnight cultures of gonococci were lyophilised, heated in lauryl sulphate with 2-mercaptoethanol, and the solubilised cell proteins were subjected to alkaline sodium dodecyl sulphate gel electrophoresis. The resulting electrophoresis patterns were scanned with a spectrophotometer, and the comparisons of the gel scans were made by superimposing pairs of scans on a view box. The gel patterns of the two scans were judged to be the same if the configuration of the peaks coincided or if in one of the two patterns a single additional peak was present in the region corresponding to a protein with an estimated molecular weight of $13000 .^{3}$

\section{ANALYSIS OF RESULTS}

Two gonococcal isolates were considered to be of the same strain if they had the same auxotypes, antibiotic susceptibility test results, and protein electrophoresis patterns. If there were discrepancies in any one of these results, the isolates were said to be of different strains. Categorical data were analysed by the $\chi^{2}$ method with Yates's correction.

\section{Results}

The number of cultures for gonococci giving a positive result were taken from paired sites as follows: cervix-rectum 41, cervix-urethra 25 , urethrarectum 1 , and cervix-rectum-urethra 2 . The auxotypes of the individual isolates were as follows: Zero 58, Pro 41, Arg 10, Arg-Hyx-Ura 21, Arg-Orn 2, Pro-Arg-Hyx-Ura 2, Arg-Orn-Hyx-Ura 2, and Pro-Arg 2. Gel electrophoresis was performed on all but nine of the 69 pairs of gonococcal isolates. One gonococcal strain was present in paired isolates from 63 of the 69 patients. Two gonococcal strains were found in the paired isolates of six of the 69 patients. The characterisation of the strains from these six patients are shown in the Table. Different gonococcal strains simultaneously infecting genitorectal sites were found in $8 \cdot 7 \%(2-15 \cdot 4 \%$, $\mathbf{9 5 \%}$ confidence interval) of the patients. Infection with more than one gonococcal strain was present in $9 \cdot 7 \%(4 / 41)$ of the cervical-rectal pairs and $8 \%$ $(2 / 25)$ of the cervical-urethral pairs. This difference was not significant when tested by the $\chi^{2}$ method. Comparisons of the protein electrophoresis patterns were at variance with the auxotyping and the antibiotic susceptibility results in only one of the pairs in this study (patient 1 , see Table). The two isolates from this patient had the same protein electrophoresis pattern despite differences in the auxotypes and the antibiotic susceptibilities.

\section{Discussion}

Other investigators have detected infections with more than one gonococcal strain in a single patient by means of differences in gonococcal antigens, gonococcal auxotypes, and the combination of auxotyping and antibiotic susceptibility testing. . $^{8-10}$ The present study may be compared only with that of Catlin and Pace, ${ }^{8}$ who used similar methods of

TABLE Results of auxotyping, gel electrophoresis, and antibiotic susceptibility tests of pairs of gonococci isolated simultaneously from rectal and genital sites in six patients

Minimum inhibitory concentration $(\mu \mathrm{g} / \mathrm{ml})$

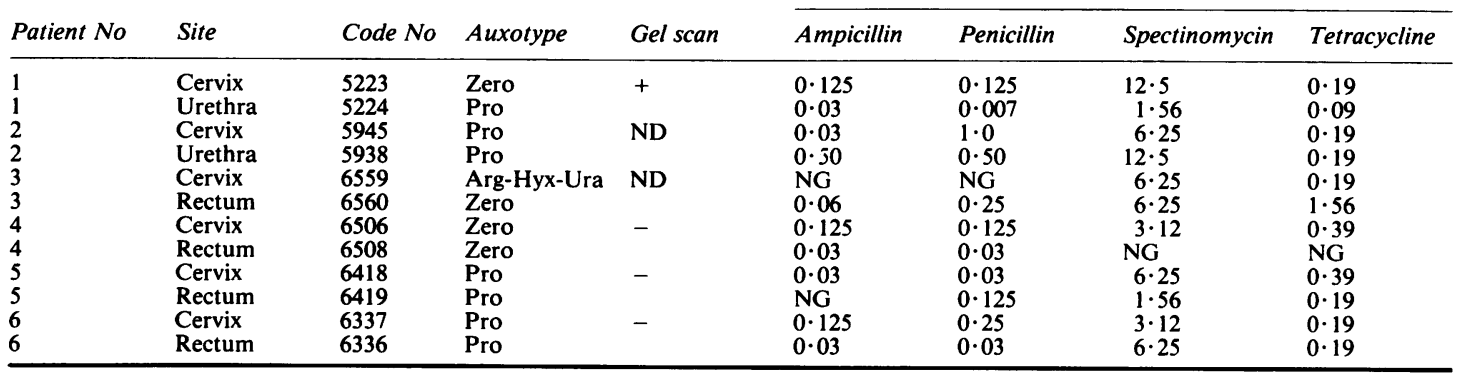

$+\quad$ Agreement between pairs

- Disagreement between pairs NG No growth ND Not done 
characterising gonococci. They identified two gonococcal strains in paired isolates of six $(8 \cdot 8 \%)$ of 68 women with infections of the cervix and rectum. However, three of the six pairs reported as different had identical auxotypes and the antibiotic susceptibility test results of these pairs differed by less than two dilutions. Thus, on the criteria of the present study, only $4 \cdot 4 \%(3 / 68)$ of the paired isolates would have been different.

Selection of multiple colonies from the same culture plate might identify more patients with infections with two or more gonococcal strains. Johnston et $a^{9}{ }^{9}$ identified gonococci with two or more serotypes in five patients by serotyping 15-20 bacterial colonies from a single culture plate. However, this study did not attempt to estimate the frequency of infection with multiple serotypes. Short et al ${ }^{10}$ examined the auxotypes of eight gonococcal colonies picked from single cultures of each of $\mathbf{4 0}$ patients. More than one gonococcal auxotype was identified in 10 patients. However, this finding may not be comparable with that of the present study since they used a different typing method from that of Catlin. ${ }^{6}$

Simultaneous infections with multiple types of a single species of a Gram-negative organism are not limited to gonococcal infections. Three or four strains of Escherichia coli may be present in the stool specimens of healthy individuals. ${ }^{11}$ Different $E$ coli serotypes were also found in $20 \%$ of paired urine and faecal cultures from 98 patients with urinary tract infections. ${ }^{12}$ Similarly, $17 \%$ of 75 patients with Salmonella infections of the intestine had multiple serotypes in their stool cultures. ${ }^{13}$

Although gonococcal infection of the urethra or rectum in women may possibly result from contact with infected vaginal secretions, infection with two or more gonococcal strains may be the result of vaginal or rectal intercourse with two or more infected sexual partners or both. Rectal intercourse was previously reported by $6 \cdot 2 \%(10 / 161)$ of women attending the clinic of the present study. ${ }^{14}$ Unfortunately, neither this study nor previous studies have detailed epidemiological data on the number or nature of sexual contacts. Thus, variations between the results of the studies mentioned above and those of the present study may reflect the frequency of sexual contact of women patients with different infected partners. Studies of gonorrhoea in women prostitutes or studies which examine a larger number of culture samples from each patient might indicate a higher percentage of patients infected with several gonococcal strains.

The author gratefully acknowledges the technical assistance of Mrs Betty R Miller, the suggestions of Dr Ernst Stolz and Dr A P Orange, Department of
Dermatology, and the advice of $\operatorname{Dr} R$ van Strik, Department of Biostatistics, Erasmus University, Rotterdam. The co-operation of Dr Philip G Weiler, Commissioner of Health, Lexington-Fayette County Health Department, made study of the gonococcal strains possible. The reference strains for the antibiotic susceptibility tests ( $N$ gonorrhoeae, F18 and F29 and Sarcinia lutea F20) were generously provided by Dr Clyde Thornsberry of the Center for Disease Control, Atlanta, Georgia. The author also thanks Dr B Wesley Catlin of the Medical College of Wisconsin for the gonococcal strains of known auxotype (Thi, Pro-Hyx, Zero, Pro-Met-ThPP). Funds for this study were provided in part by a grant from the National Institutes of Allergy and Infectious Diseases, AI-11304, and by a grant from the Alexandrine and Alexander Sinsheimer Fund.

\section{References}

1. Schmale JD, Martin JE, Domescik G. Observations on the culture diagnosis of gonorrhea in women. JAMA 1969; 210: 312-4.

2. Rein MF, Jaffe HW, Reynolds GH, Thornsberry C, Zaidi A, Wiesner PJ. Rectal gonococcal infection in women. Abstract No. 80. American College of Physicians 57th Annual Session, Philadelphia $\mathrm{Pa}, 1976$.

3. Noble RC, Schell SC. Acrylamide gel electrophoresis of proteins of Neisseria gonorrhoeae as an epidemiologic tool. Infect Immun 1978; 19: 178-86.

4. Kellogg DS, Peacock WL, Deacon WE, Brown L, Pirkle CI. Neisseria gonorrhoeae I Virulence genetically linked to clonal variation. J Bacteriol 1963; 85: 1274-9.

5. Jaffe HW, Biddle JW, Thornsberry C, et al. National gonorrhea therapy monitoring study. In vitro antibiotic susceptibility and its correlation with treatment results. N Engl J Med 1976; 294: 5-9.

6. Catlin BW. Nutritional profiles of Neisseria gonorrhoeae, Neisseria meningitidis, and Neisseria lactamica in chemically defined media and the use of growth requirements for gonococcal typing. J Infect Dis 1973; 128: 178-94.

7. Carifo K, Catlin BW. Neisseria gonorrhoeae auxotyping. Differentiation of clinical isolates based on growth responses on chemically defined media. Appl Microbiol 1973; 26: 223-30.

8. Catlin BW, Pace PJ. Auxotypes and penicillin susceptibilities of Neisseria gonorrhoeae isolated from patients with gonorrhoea involving two or more sites. Antimicrob Agents Chemother 1977; 12: 147-56.

9. Johnston KH, Holmes KK, Gotschlich EC. The serological classification of Neisseria gonorrhoeae. I Isolation of the outer membrane complex responsible for serotypic specificity. $J$ Exp Med 1976; 143: 741-57.

10. Short HB, Ploscowe VB, Weiss JA, Young FE. Rapid method for auxotyping multiple strains of Neisseria gonorrhoeae. $J$ Clin Microbiol 1977; 6: 244-8.

11. Sears HJ, Brownlee I, Uchiyama JK. Persistence of individual strains of Escherichia coli in the intestinal tract of man. $J$ Bacteriol 1950; 59: 293-301.

12. Vosti KL, Goldberg LM, Monto AS, Rantz LA. Host-parasite interaction in patients with infections due to Escherichia coli I The serogrouping of $E$ coli from intestinal and extraintestinal sources. J Clin Invest 1964; 43: 2377-85.

13. Juenker AP. Infections with multiple types of Salmonella. Am J Clin Pathol 1957; 27: 646-51.

14. Noble RC, Kirk NM, Slagel WA, Vance BJ, Somes GW. Recidivism among patients with gonococcal infection presenting to a venereal disease clinic. Sex Transm Dis 1977; 4: 39-43. 